Revista de Ciencias Sociales - Número 65 (2014) - Páginas 121-146

Pragmatismo y derecho: notas para un enfoque científico en la teoría...

\title{
PRAGMATISMO Y DERECHO: NOTAS PARA UN ENFOQUE CIENTÍFICO EN LA TEORÍA JURÍDICA DEL SIGLO XXI
}

\author{
PRAGMATISM AND LAW: NOTES FOR A SCIENTIFIC \\ THESIS ON 21ST CENTURY LEGAL THEORY
}

\author{
NICOLÁS LÓPEZ PÉREZ* \\ Departamento de Ciencias del Derecho \\ Facultad de Derecho, Universidad de Chile \\ nicolopez@ug.uchile.cl
}

\section{Resumen}

El presente trabajo pretende analizar la aproximación pragmatista sobre el derecho de Jules Coleman, con el objeto de situarla como uno de los enfoques científicos en la teoría del derecho del siglo XXI, respecto del estado del arte de la pregunta de "¿qué es el derecho?". Para ello dispondré de dos ejes temáticos. El primero, abordará los elementos filosóficos que moldean el planteamiento de Coleman como un pragmatismo jurídico concreto y lo comparará con los otros pragmatismos jurídicos que se mezclan con las tesis

* Parte de las ideas de este trabajo fueron discutidas en el IV Coloquio Internacional de la Sociedad Chilena de Filosofía Analítica (25-28 de Marzo de 2014) bajo el nombre de "Pragmatismo, naturalismo y filosofía jurídica contemporánea: el regreso de la concepción cientificista en la reflexión del derecho”. Agradezco las observaciones de los asistentes al encuentro, particularmente del Dr. José Tomás Alvarado y de Ezequiel Monti. Artículo recibido el 3 de junio de 2014 y aceptado el 22 de octubre de 2014.

Revista de Ciencias Sociales - Número 65 (2014) - Universidad de Valparáíso - ISSN 0716-7725-Valparaíso, Chile 
de los realistas jurídicos norteamericanos. El segundo, examinará su carácter científico, y lo ubicará en la filosofía analítica del derecho de este siglo en relación a las otras tesis fuertes sobre el derecho.

\section{Palabras claves} jurídica.

Pragmatismo, Filosofía analítica, Jules Coleman, Cientificismo, Teoría

\section{Abstract}

This paper will analyze Jules Coleman's pragmatic approach on the Law, in order to place it as one of the scientific approaches in the $21^{\text {st }}$ century legal theory, about the state of the art of the question "what is Law?" To this I will put two themes. The first will address the philosophical elements that shape the approach of Coleman as a specific legal pragmatism and compare it with other legal pragmatism that blend with the thesis of American legal realists. Second, examine its scientific character, and place it in the analytic philosophy of law of this century in relation to the other strong thesis about the Law.

\section{Keywords}

Pragmatism, Analytical philosophy, Jules Coleman, Scientism, Jurisprudence.

"Si se quiere saber lo que hace del derecho lo que es, o de $X$ lo que es, se necesita saber de qué manera los $X$ son descritos y explicados por las ciencias".

Brian Leiter

\section{INTRODUCCIÓN: PRAGMATISMO Y TEORÍA JURÍDICA}

A lo largo del siglo XX se dio paulatinamente la extensión de las discusiones en filosofía pura al interior de la tradición analítica hacia el espectro de la filosofía aplicada en la misma. Pasa en el caso, entre otros, de la mente, el lenguaje, la ciencia y el derecho. Este último se ha alimentado considerablemente de los debates filosóficos en la tradición, principalmente por la contienda entre Russell y Strawson, que fue

Facultad de Derecho y Ciencias Sociales - Universidad de Valparaíso - Chile 
absorbida como insumo por H. L. A. Hart en la gestación de The Concept of Law (1961), texto en el cual se intentó elucidar al concepto de derecho y se identificó a la normatividad con nuestras maneras de hablar y nuestras prácticas sociales. Postulo que dicha obra se posiciona como el centro de la teoría jurídica analítica de cara a los debates posteriores, incluso a los actuales. Con su publicación, el enfoque de la teoría del derecho excluía a la vereda cientificista del plano erudito. Se había olvidado a su último bastión, la Reine Rechtslehre de Hans Kelsen, que no era más que un intento de explicar qué es el derecho excluyendo cualquier elemento que desembarazara su análisis. Un proyecto que no estaba interesado en describir cuenta del fenómeno jurídico, sino que pretendía formar un conjunto lógico donde fuera posible cuantificar la realidad en la que estaba inmerso al derecho y así también, a este, como un sistema in toto.

El mapa filosófico sería desbordado de lo que era antes: el continente y el archipiélago del conocimiento, y se extendería hacia Norteamérica. Así, para la filosofía analítica del derecho, dos hitos importantes: los guiños de los realistas jurídicos norteamericanos a lo largo de la primera mitad del siglo XX y la publicación de Two Dogmas of Empiricism en 1952. Este último, atisbaría la debilidad de la distinción analítico-sintético y el reductivismo, los llamados "dogmas del empirismo" sobre los cuales la filosofía en general, se cimentaba, especialmente lo que provenía de las elucubraciones del Círculo de Viena. Quine con ello estaría dándole un nuevo respiro al empirismo y a la concepción cientificista, debilitada por On Referring (1950) en el clásico trazo de la tradición analítica hecho por On Denoting (1905).

La tesis de Quine se proyectaría por un tiempo en la filosofía analítica, hasta la controversia que iniciaría Hilary Putnam en su The Collapse of the Dichotomy Fact/Value. Este plantearía que no es de la exclusiva propiedad de los juicios ser analítico o sintético, sino que el punto de partida es ser analítico y luego, verificado de la mejor manera posible. Con ello, acuño la existencia de un debate, el que llamaré "Quine-Putnam", que posteriormente nutriría la reflexión del derecho.

A simple vista, esta discusión sería recogida en los planteamientos de Brian Leiter y de Jules Coleman. El primero, propondría en 2001, el giro naturalista en la filosofía del derecho, aduciendo la superación del 
giro lingüístico que caracteriza a la tradición analítica, donde la mayoría de los problemas filosóficos ya habrían dejado de ser problemas del lenguaje (a priori) y debían ser juzgados ante el tribunal de la ciencia natural, o sea, pasar por el filtrado a posteriori, dándose así un proceso de naturalización de la filosofía como lo planteó Quine en 1969. La novedad es que Leiter propuso que la mayoría de las filosofías aplicadas superaron el giro lingüístico y que la filosofía del derecho debía seguir los pasos de las otras disciplinas, por lo que la jurisprudence se naturaliza ${ }^{1}$. Con ello también, se permite señalar que el análisis conceptual que subyace al texto de Hart, no es un método idóneo para medir y/o dar cuenta de lo que es el derecho y cómo opera. Coleman, el mismo año que Leiter propuso la descrita tesis, expuso en The Practice of Principle que las reglas poseen una explicación fáctica, así como su lenguaje y el contenido del derecho, que no son más que parte de una maquinaria pragmática, sujeta a ciertos criterios prácticos. El intento de una teoría pragmatista del derecho al alero de un paradigma realista, como lo es el derecho de daños (tort law).

El presente trabajo se centrará en la propuesta de Coleman, como la construcción de un enfoque científico en la teoría jurídica del siglo XXI. Un planteamiento "científico" stricto sensu de lo que es el derecho, luego de 1961 fue dejado a la deriva no sólo con la lucidez y pomposidad del libro de Hart y su tesis del análisis conceptual para dar cuenta de lo jurídico y los problemas acerca del lenguaje y el derecho, sino que con la exclusión de ciertos participantes en la discusión contemporánea en teoría analítica del derecho. Para ello dispondré de dos ejes temáticos. El primero, abordará los elementos filosóficos que moldean el planteamiento de Coleman como un pragmatismo jurídico concreto y lo comparará con los otros pragmatismos jurídicos que se mezclan con los realistas jurídicos norteamericanos. El segundo, examinará su carácter científico, y lo ubicará en la filosofía analítica del derecho de este siglo en relación a las otras tesis fuertes sobre el derecho.

1. LÓPEZ PÉREZ, Nicolás: "El naturalismo jurídico como teoría del derecho: red filosófica y conceptual de un cientificismo revisitado”. En: Derecho y Humanidades, 22, 2013, en prensa.

Facultad de Derecho y Ciencias Sociales - Universidad de Valparaíso - Chile 
Pragmatismo y derecho: notas para un enfoque científico en la teoría...

\section{HACIA UNA APROXIMACIÓN PRAGMÁTICA JURÍDICA}

\section{Antecedentes histórico-filosóficos: pragmatismo en general}

El pragmatismo tiene un desarrollo no en el archipiélago "del conocimiento", sino que se origina con anterioridad, incluso, a la tesis que marca el inicio de la tradición analítica en filosofía. Aquella, es el trabajo de Frege de 1879, la Begriffsschrift (Conceptografía) ${ }^{2}$, pues ya en 1870, C. S. Pierce y William James acuñarían el término para referirse al método para clarificar la estructura de pensamiento y los contenidos de las hipótesis, explicando las posibles consecuencias de un proceder o de otro. En los trabajos de los mencionados filósofos, el fuerte estaba en el concepto de verdad ${ }^{3}$. Con posterioridad a los clásicos (Pierce y James) se desarrollaría en forma silenciosa hasta la segunda década del siglo $\mathrm{XX}$, donde tendría una caída. Luego, sería rediscutido bajo el "giro

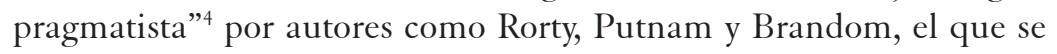
proyecta hasta hoy, inclusive.

Hoy en día el término "pragmático" está asociado con la aproximación de las cuestiones de hecho para la solución de problemas. Se dice comúnmente que, aquel que es pragmatista, es una persona que "obtiene resultados". El pragmatista se ocupa de asuntos prácticos

2. ORELLANA BENADO, M. E.: "Tradiciones y concepciones en filosofía". En: NUDLER, O. (ed.): Filosofía de la Filosofía. Enciclopedia Iberoamericana de Filosofía, 31. Trotta, Madrid, 2010. Págs. 49-78.

3. HOOKWAY, Christopher: "Pragmatism", The Stanford Encyclopedia of Philosophy, 2008. Disponible en: http://plato.stanford.edu/entries/ pragmatism/ Fecha última consulta: 18 de marzo de 2014.

4. BERNSTEIN, Richard J.: The Pragmatic Turn. Cambridge University Press, Cambridge, 2010. Ello también reconocería la idea de "los nuevos pragmatistas”, para ello véase BACON, Michael: Pragmatism. Polity Press, Oxford, 2012. Págs. 9-14; HAACK, Susan: Pragmatism: Old and New. Prometheus Books, Nueva York, 2006 o MISAK, Cheryl (ed.): New Pragmatists. Oxford University Press, Oxford, 2009.

5. BACON, M., ob. cit. Pág. 1.

Revista de Ciencias Sociales - Número 65 (2014) - Universidad de Valparáíso - ISSN 0716-7725-Valparaíso, Chile 
y siempre considerando que las respuestas a las preguntas sobre el mundo o filosóficas, ofrezcan consecuencias que sean útiles para nuestras vidas. La investigación sobre la naturaleza de la verdad, cómo el conocimiento es posible, qué ocurre con una vida moral y política, deben ser direccionadas en el camino de lo verdadero ${ }^{6}$.

La idea del pragmatismo como una forma de filosofía inmediatamente suscita preguntas, así como en líneas precedentes, este desarrollo que tuvo de forma paralela (y a la vez, distinta) de los analíticos duros. El concepto "pragmatismo" si bien, nace como tal de una lección de William James en 1898, proviene de un ensayo de C. S. Peirce de 1878 llamado, How to Make Our Ideas Clear. En él, explica que se tiene una tendencia a justificar los pensamientos a partir de cuestiones tangibles y prácticas, con el objeto de escoger las mejores explicaciones ${ }^{7}$. El pragmatismo es fuerte en la primera mitad del siglo $\mathrm{XX}$ en Estados Unidos, aunque los representantes de la “vieja” escuela como Dewey, Peirce y James discurrían entre sí. A medida que iban falleciendo, las enseñanzas pragmatistas se iban quedando en el aire, hasta, según Bacon, 1979 con la publicación de la primera edición de Philosophy and the Mirror of Nature de Richard Rorty ${ }^{8}$. Luego añade, Bacon que el eclipse del pragmatismo a fines de la primera mitad del siglo XX estuvo marcado por el positivismo lógico, reinante en el mapa epistémico europeo. Parte de lo que es recogido por los naturalistas en los años 50 en Estados Unidos, fundamentalmente Quine y Sellars, quienes mezclaron parte de los antecedentes filosóficos de sus redes institucionales en Norteamérica con parte del intercambio recibido por filósofos europeos.

Cuando se piensa en pragmatismo, debido a la disociación de planteamientos existentes entre los "viejos" pragmatistas, se debe ser

6. JAMES, William: Pragmatism. Hackett Publishing, Indianapolis (IN), 1977. Pág. 347.

7. PEIRCE, C. S.: The Essential Peirce (eds. Houser, N. y Kloerel, C.). Indiana University Press, Bloomington (IN), 1992. Pag. 131.

8. BACON, M., ob. cit. Pág. 7.

Facultad de Derecho y Ciencias Sociales - Universidad de Valparaíso - Chile 
cauteloso a la hora de elaborar un estudio metafilosófico de la tradición pragmatista'. Se dice que ab initio hubieron dos vertientes del pragmatismo, una por el lado de Peirce y otra por el lado de James. Sobre la primera nacen autores como Putnam (útil para el análisis en teoría del derecho) y Haack. En la otra vereda, se encuentra Rorty.

En el pragmatismo de Putnam, es relevante destacar - para efectos de este trabajo- que la selección de teorías siempre presupone valores, y que es una sucesión natural del "progreso de la ciencia" y la efectividad de una teoría el hacerlo. Asimismo, quienes han elucubrado sus planteamientos sobre lo anterior, al igual que los empiristas, estos filósofos están determinados a mantener los ojos cerrados ante el hecho de que la ciencia física presupone juicios sobre coherencia, simplicidad (que no es un único 'parámetro', sino que engloba distintos valores), belleza, naturalidad y demás, muchos autores contemporáneos que se refieren a los valores como puramente 'subjetivos', y a la ciencia como puramente 'objetiva', siguen con los ojos cerrados ante el mismo hecho. Y, sin embargo, la coherencia, la simplicidad y similares son valores. Putnam llama a dejar de pensar en el concepto de 'valor' como sinónimo de una cuestión 'ética', máxime si la ciencia presupone valores, los llamados epistémicos ${ }^{10}$.

El valor y la normatividad permean la totalidad de la experiencia. También aquello que se considera ciencia está determinado por las creencias de lo que según las personas debería ser la ciencia (valores tales como simpleza, belleza científica, etc.). La distinción de Putnam entre los valores es más cercana a una tendencia pragmática de rechazar la existencia de verdades absolutas; de expresar que las ideas son provisionales y están sujetas al cambio, a la luz de la investigación futura.

Una aproximación pragmatista en la teoría del derecho podría ayudarnos a describir cuál teoría es mejor para dar cuenta del fenómeno jurídico en la realidad o bien, cuál es el que mejor lo describe, pero no nos dejemos engañar sin oír todo el argumento.

9. RESCHER, Nicholas. Realistic Pragmatism: An introduction to pragmatic philosophy. State University of New York, Nueva York, 2000.

10. PUTNAM, Hilary: El desplome de la dicotomía hecho-valor y otros ensayos. Paidós, Barcelona, 2001 (trad. Francesc Forn i Argimon). Pág. 46.

Revista de Ciencias Sociales - Número 65 (2014) - Universidad de Valparáíso - ISSN 0716-7725-Valparáiso, Chile 


\section{Para un pragmatismo jurídico en concreto}

En The Practice of Principle, Coleman si bien va en la línea de Peirce y Putnam, reconoce como influencias filosóficas a Sellars, Quine y Davidson, no obstante, su propósito con esta aproximación no radica en una reivindicación de las tesis de los anteriores, sino más bien como un aprendizaje con respecto del estudio del derecho realizado en otros trabajos suyos ${ }^{11}$ bajo la etiqueta de "pragmatista". Es tal aquello, pues casi nada de los trabajos de la teoría jurídica contemporánea norteamericana que han utilizado ese término han llevado a cabo una empresa filosófica en términos estrictamente pragmatistas. Asimismo, se han posicionado, indistintamente, como enfoques norteamericanos a la filosofía analítica ${ }^{12}$.

Sobre lo anterior, "pragmatismo" parece ser una expresión familiar entre los juristas norteamericanos (los autores del realismo jurídico de esta raigambre), a diferencia de lo que ocurre con "naturalismo". Al igual que Coleman, Leiter afirma que la trivialidad con que la etiqueta ha sido usada, ha llevado a malos entendidos ${ }^{13}$. Respecto a esto, Singer retrata lo siguiente:

"Los realistas jurídicos querían reemplazar el formalismo con una actitud pragmática hacia el derecho en general. Esta actitud trata al derecho como algo hecho, no descubierto. Entonces el derecho es, y debe ser, basado en la experiencia humana, en la política y en la ética, más que en la lógica formal" ${ }^{14}$.

11. Por ejemplo, COLEMAN, Jules: Risks and Wrongs. Cambridge University Press, Cambridge, 1992; "Negative and Positive Positivism”. En: Cohen, M.

(ed.): Ronald Dworkin and Contemporary Jurisprudence. Duckworth, Londres, 1983. Págs. 28-48.

12. COLEMAN, Jules: The Practice of Principle. In Defence of a Pragmatist Approach to Legal Theory. Oxford University Press, Oxford, 2001. Pág. vii.

13. LEITER, Brian: Naturalismo y teoría del derecho. Marcial Pons, Madrid, 2012 (trad. Giovanni Battista Ratti). Pág. 84.

14. SINGER, Joseph: "Legal Realism Now”. En: California Law Review, 76, 1988. Pág. 474.

Facultad de Derecho y Ciencias Sociales - Universidad de Valparaíso - Chile 
Pragmatismo y derecho: notas para un enfoque científico en la teoría...

Según Leiter, la anterior, no parece una caracterización del pragmatismo, en el sentido estrictamente filosófico. El pragmatismo posee un doble compromiso, que concierne a la empresa de teorizar y a la epistemología ${ }^{15}$. Así el criterio pragmático ayuda a la exitosa construcción de una teoría, que pretende mostrar un determinado juego de lenguaje o realidad o incluso, aún que se conozcan tales ${ }^{16}$.

Luego, el pragmatismo está preocupado de la aceptabilidad de las creencias y al mismo tiempo, sobre las aceptables se discute. En primer lugar, existen aquellas que deben satisfacer ciertos requisitos epistémicos (correspondencia con la realidad; estar garantizadas bajo condiciones epistémicas ideales; aparecer en nuestra mejor reconstrucción científica del mundo; ser inferidas de algún conjunto de creencias fundamentales). En segundo lugar, aquellas que deben ser aceptadas por el criterio a posteriori de utilidad para particulares propósitos humanos, esto es, que funcionan en relación a un sistema práctico. Sobre ello, el pragmatismo no niega las distinciones, las definiciones, la coherencia, los argumentos abstractos o las construcciones teóricas, sino que las suma a la pregunta de si son útiles para los fines humanos; en determinadas investigaciones científicas, el tema se trata de una manera cerrada ${ }^{17}$.

Para aceptar normas epistémicas (las que indican que es preciso creer) se requiere de criterios pragmáticos, vale decir, tenemos que aceptar las normas epistémicas que funcionan para nosotros (que nos ayudan en predecir las experiencias sensoriales, que nos permiten manipular y controlar el medioambiente de manera exitosa, que nos consienten en operar al mundo). Estos criterios, en definitiva, son los únicos posibles para la aceptación de estas normas, pues no es posible defender nuestra elección de una norma epistémica cualquiera sobre

15. LEITER, B. Naturalismo y teoría ... ob. cit. Pág. 86.

16. Un argumento desarrollado en WARNER, R.: "Why Pragmatism? The Puzzling Place of Pragmatism in Critical Theory”. En: University of Illinois Law Review, 1993. Pag. 535-63 y en HAACK, Susan: "Pragmatism”. En: Dancy, J. y Sosa, E. (eds.): A Companion to Epistemology. Blackwell, Oxford, 1992, Págs. 351-7.

17. LEITER, B. Naturalismo y teoría ... ob. cit. Pág. 87.

Revista de Ciencias Sociales - Número 65 (2014) - Universidad de Valparáíso - ISSN 0716-7725-Valparaíso, Chile 
un basamento epistémico, pues ello formula una cadena centrípeta ad infinitum. Leiter, luego, se pregunta: ¿qué normas funcionan efectivamente para nosotros? Para contestarlo se apoya en el ejemplo: "No creas en una hipótesis que aparece en una explicación de la experiencia que no sea consiliente", parece ser una regla acerca del conocimiento (consilience rule). Una explicación que no es consiliente es la que ofrece lo que proporciona la explicación, ello es restringido en relación con el evento a explicar ${ }^{18}$. Así es como el criterio pragmático opera $^{19}$. Supongamos, en esta línea, que es de noche y estamos durmiendo en nuestras casas y de pronto, la tierra comienza a moverse y el librero se cae. ¿Qué hecho del mundo podría explicar este suceso? Intentemos dos hipótesis:

a) En la casa hay fantasmas que comenzaron a remecer la casa con el fin de asustar y con ello, derribaron el librero.

b) Hubo un terremoto de considerable magnitud en una zona cercana a la casa.

La regla de la consiliencia, junto al resto de normas de la epistemología científica han sido útiles para explicar la no existencia se seres sobrenaturales, y con ello, han constituido una suerte de bagaje en la práctica científica, aquella que ha podido posibilitar una serie de cosas, como, que el ser humano tenga jaquecas, que los submarinos puedan andar bajo el mar o incluso, que un pequeño cilindro de carbono pueda generar energía. Lo anterior toma la idea quineana de que la ciencia es sentido común autoconsciente y que las demás disciplinas siguen un continuum con ella, que Leiter apoya. A ello, el pragmatismo cae en esa red argumentativa, donde la preferencia por la ciencia se cubre en virtud de que es una explicación útil y práctica para los fenómenos naturales, así también, ofrece respuesta a los asuntos acerca del mundo. Quine, ya lo decía, la ciencia constituye el paradigma del genuino conocimiento ${ }^{20}$.

18. THAGARD, P.: “The Best Explanation: Criteria for Theory Choice”. En: Journal of Philosophy, 75(2), 1978. Págs. 79-85.

19. LEITER, B. Naturalismo y teoría ... ob. cit. Págs. 88-9.

20. QUINE, W. V.: The Ways of Paradox and Other Essays. Harvard University Press, Cambridge (MA), 1976. Pág. 233.

Facultad de Derecho y Ciencias Sociales - Universidad de Valparaíso - Chile 
Con todo, la predicción en una teoría pragmatista, existe. Leiter y Coleman han observado a los realistas jurídicos norteamericanos como pragmatistas. El primero postula sobre estos teóricos del derecho que "la construcción de teorías filosófico-jurídicas está vinculada a un valor práctico, es decir, debe hacer posible predecir lo que harán los tribunales"21. En este punto, Mackie y Dworkin discuten. El primero, se posiciona en la vereda de los realistas, defendiendo que lo importante es lo que los jueces hacen efectivamente, no así lo que dicen que hacen o lo que piensan que hacen ${ }^{22}$. Respecto a Dworkin, la explicación teórica debe radicar en lo que los jueces dicen que hacen y lo que piensan que hacen ${ }^{23}$.

Fuera de esos alegatos que se proyectan en una teoría de la adjudicación, cuestión que inconscientemente consiguen los realistas jurídicos norteamericanos, Coleman explica que el derecho es una práctica normativa ${ }^{24}$. Instantáneamente sería posible hacer el parangón entre la propuesta de este filósofo con el positivismo jurídico, inclusive, preguntarnos a priori, si Coleman es un positivista o no ${ }^{25}$.

El punto de partida de las teorías sobre el derecho o su naturaleza descansa en The Concept of Law, así como la disputa acerca de métodos y propósitos en la filosofía (analítica) del derecho. La propuesta de Hart se desglosa en la sociología descriptiva y el análisis conceptual. Este último es el que suscita las discusiones en todas direcciones, en la sección siguiente veremos con detalle. Por ahora, resta introducir la manera en

21. LEITER, B. Naturalismo y teoría ... ob. cit. Pág. 91.

22. MACKIE, J. L. “The Third Theory of Law”. En: Cohen, M. (ed.): Ronald Dworkin and Contemporary Jurisprudence. Duckworth, Londres, 1983. Págs. 161-70.

23. DWORKIN, Ronald: Taking Rights Seriously. Harvard University Press, Cambridge (MA), 1977. Págs. 22 y 112.

24. COLEMAN, J. The Practice of Principle... ob. cit. Pág. xviii.

25. ZIPURSKY, Benjamin: "Pragmatism, Positivism, and the Conventionalistic Fallacy”. En: Campbell, J. K., O’Rourke, M. y Shier, D. (eds.): Law and Social Justice. The MIT Press, Cambridge (MA), 2005.

Revista de Ciencias Sociales - Número 65 (2014) - Universidad de Valparáíso - ISSN 0716-7725-Valparaíso, Chile 
como Coleman entra a la discusión sobre metodología de la teoría del derecho con una aproximación pragmática. Dice él,

“(e)l positivismo jurídico es una teoría general del derecho que afirma que la moral no es una condición necesaria de la legalidad o de lo que sea que sea el derecho, el criterio de la legalidad es convencional. El positivismo jurídico no niega que el derecho de una comunidad, parcial o totalmente, pueda ser moralmente defendido o de otra manera, pueda expresar o cobijar ideales morales (...) una teoría de la naturaleza del derecho o del concepto del mismo es una teoría acerca de lo que tiene que ser un cierto tipo de práctica social. No es una teoría acerca de qué particulares cuerpos sustantivos de la ley deberían lucir como o menos, con razón debemos tener un derecho de daños (tort), de contratos (contract) o penal (criminal)"26.

A grandes rasgos, la oferta de la tesis de Coleman se asoma afirmando que una teoría que indague en la naturaleza o el concepto del derecho se posiciona en lo que sea una determinada práctica social o lo mismo, una cuestión de hecho. Luego, allí es donde se encuentra una propuesta pragmatista. Sugiero ser cauteloso con esta primera aproximación.

A continuación expresa los dos grandes desafíos que el estado del arte le impone al análisis conceptual de cara a la filosofía jurídica analítica del siglo XXI. El primero se refiere a que debemos renunciar al análisis conceptual a priori totalmente, abandonando a la teoría jurídica analítica en favor de una teoría jurídica naturalizada o científica ${ }^{27}$. Asimismo, el análisis conceptual, bajo esta rúbrica, es incapaz en sus propios términos de proveer una comprensión de la

26. COLEMAN, J. The Practice of Principle... ob. cit. Pág. xix.

27. Una contraposición entre un análisis conceptual y un análisis cientificista del derecho, todo en las fauces de los intentos teóricos para dar cuenta de lo jurídico. Este desafío le coloca a Hart y sus sucesores, nuevamente en el camino a una reflexión cientificista sobre el derecho.

Facultad de Derecho y Ciencias Sociales - Universidad de Valparaíso - Chile 
práctica jurídica o bien, la resolución de los debates metodológicos que dividen a los teóricos del derecho. El segundo, se refiere a que el análisis conceptual en su faceta de ser una investigación filosófica sobre la naturaleza del derecho es desde el inicio y hasta el fin, un ejercicio en teoría política sustantiva. Ese es el cortafuego que Dworkin ha colocado desde el inicio del debate con Hart, por 1967 con Is Law a System of Rules?

Coleman intenta tomar partido por una posición que no esté en el extremo de una teoría naturalizada o científica y al mismo tiempo, una que no se case con una cuestión política. En ningún caso, acepta como un método propio para dar cuenta del derecho, al análisis conceptual. La propuesta de Coleman respecto de un pragmatismo (jurídico) stricto sensu, bien se dijo, comienza por disipar las otras teorizaciones sobre el derecho que se hacían llamar pragmáticas, o se "pragmatismo jurídico".

Para avanzar a un método pragmático en teoría del derecho, es necesario distinguir entre explicaciones teóricas y justificaciones teóricas. Ambas pueden iluminar o profundizar nuestro entendimiento; las explicaciones lo hacen contándonos qué es la naturaleza de un objeto o bien, por qué las cosas son como son; en contraste, las justificaciones buscan defender o legitimar ciertos tipos de cosas, por ejemplo, acciones, reglas, cursos de conductas, prácticas, entre otros. Las explicaciones están reguladas por normas de precisión descriptiva y/o predictiva, mientras que las justificaciones lo están por las apropiadas normas morales ${ }^{28}$. Esta prevención puede caer en la distinción de Putnam entre valores epistémicos y valores éticos. Ambos, quizás, podrían ayudar en la búsqueda de un concepto de derecho, sin embargo, el desacuerdo podría recaer - como ocurre en la literatura actual — sobre cuáles valorarían, cuáles evaluarían, cuáles describirían y cuáles medirían.

Por ello, con la aclamada tesis de Hart sobre el concepto de derecho surgen al paso, cuestiones como un concepto o bien, nuestro concepto. Los pragmatistas, digamos, se inclinan por un concepto, aunque eso podría ser engañoso si se piensa en la consiliencia; he ahí

28. COLEMAN, J. The Practice of Principle... ob. cit. Pág. 3.

Revista de Ciencias Sociales - Número 65 (2014) - Universidad de Valparáíso - ISSN 0716-7725-Valparaíso, Chile 
una diferencia entre el pragmatismo de Coleman y el que puede emerger de los realistas jurídicos norteamericanos.

Ahora bien, Coleman distingue como punto de partida las afirmaciones: (i) "nuestro concepto de $\mathrm{x}$ depende en qué selo que debería ser ese concepto" de (ii) "nuestro concepto de $\mathrm{x}$ depende en lo que $\mathrm{x}$ debería ser”. Respecto del derecho, (i) podría implicar que nuestro concepto del derecho responda a las normas que gobiernen la formación de los conceptos y la construcción de las teorías (normas teoréticas o normas epistémicas) y ello es revisable a la luz de las consideraciones normativas de esa clase. (ii) podría implicar que nuestro concepto del derecho responda a normas morales o políticas en la forma que haga que el análisis de ese concepto, primordialmente sea parte de un sustantivo argumento político o moral. Según Coleman, ciertamente nuestro concepto del derecho depende en parte de qué debería ser nuestro concepto del derecho, pero este mismo no es, en ninguna forma teóricamente interesante, dependiente de lo que el derecho debe ser. Una aproximación pragmatista al derecho debiera preocuparse que el asunto sea de carácter epistémico o teórico, no moral ni menos político ${ }^{29}$.

Propiamente no hay un intento real de pragmatismo jurídico, un punto en el que concuerdo con Coleman. Junto a esto, el término parece bien vilipendiado y poco entendido, por lo que los antecedentes filosóficos y la construcción de un bagaje teórico es bien magro. Coleman elabora un enfoque pragmatista del derecho, que en su aplicación práctica tiene acogida en el tort law (derecho de daños) ${ }^{30}$, que tiene cinco pilares que le dan su base ${ }^{31}$ :

1. Un compromiso con el no-atomismo semántico, el que afirma que las condiciones de aplicación de un concepto (o de la palabra que lo expresa) y el valor veritativo de una creencia en el cual el concepto figura (o la proposición que expresa

29. Ibíd., Págs. 3-4.

30. COLEMAN, Jules: “Los fundamentos del bienestar". En: Derecho y Humanidades, 10, 2004. Págs. 17-46.

31. COLEMAN, J. The Practice of Principle... ob. cit. Págs. 6-10.

Facultad de Derecho y Ciencias Sociales - Universidad de Valparaíso - Chile 
como una creencia) depende al menos de algunas de las relaciones entre ese concepto o palabra y otros conceptos y palabras.

2. El contenido de un concepto puede ser analizado en términos del rol inferencial que juega en la variedad de prácticas en las cuales figura. Los roles inferenciales que nuestros conceptos juegan, revela la red de relaciones holísticas (o semiholísticas) en las que descansa uno respecto de otro, y es esa red, la que determina el contenido de un concepto.

3. El pragmatismo de Coleman reconoce la explicación por encarnación como una forma legítima de explicación filosófica de una práctica, esto quiere decir que en ciertas clases de prácticas, los roles inferenciales de los conceptos pueden ser vistos para salir juntos en la forma en que reflejen un principio general.

4. El significado de un concepto en cualquier práctica influencia su propio significado en todas las otras.

5. Un compromiso con la revisión (o su posibilidad) de todas las creencias es un sello distintivo de la actitud pragmática. Todos están de acuerdo que las creencias empíricas son revisables y deben serlo, a la luz de la recalcitrante experiencia.

Acto seguido, Coleman traza la distinción entre criterios de revisión pragmáticos y empíricos. Los primeros incluyen algunas preocupaciones teoréticas como por ejemplo, la coherencia, la simplicidad y la consiliencia, junto a consideraciones de utilidad práctica $^{32}$. La tesis de Coleman extiende la revisión (o su posibilidad) a una "red de creencias" para incluir las categorías y conceptos que empleamos para organizar nuestro pensamiento acerca de varias áreas del derecho así como las categorías que empleamos para dividir un área

32. Coleman aquí, siguiendo la lógica de la distinción analítico-sintético, explica que los enunciados sintéticos son revisables a la luz de la recalcitrante experiencia. Mientras que los analíticos (inmunes a la desconfirmación por medio de la experiencia) son revisables solo a la luz de las incumbencias pragmáticas. Se acepta, en principio que todos los enunciados son en principio revisables por ambos tipos de criterios (2001: 9).

Revista de Ciencias Sociales - Número 65 (2014) - Universidad de Valparáíso - ISSN 0716-7725-Valparaíso, Chile 
del derecho de otra. Todas son, en principio, revisables ${ }^{33}$. Una tesis pragmatista como la de Coleman puede ser útil para reconocer cuestiones dogmáticas o de la ordenación respecto de las ramas del derecho, un intento insatisfactorio en $\operatorname{Ross}^{34}$, que puede aclarar la gran red de conceptos jurídicos, luego de la elucidación o fijación de una solución a la pregunta de "¿qué es el derecho?".

Una de las ventajas de la aproximación pragmatista a la teoría del derecho de Coleman es que permite la adopción de conceptos locales, por lo que la idea de un concepto de derecho queda satisfecha, bajo la premisa de que aquél posee un determinado valor epistémico. Ahora bien, para saber si ello es consecuente con el edificio que construyó con sus planteamientos, es necesario pensar, por ejemplo, en el caso del tort law. Como un concepto de derecho, para explicar lo que ocurre con la responsabilidad extracontractual, (1) queda satisfecho en el respecto de, por ejemplo, "el sujeto A que manejaba un auto, choca intencionalmente a un sujeto B que iba en bicicleta". La regla en el sistema determina que en esta situación se aplica el concepto de "dolo", luego éste tendrá un valor de verdad, dependiendo del juego que exista al interior del sistema. Una especie de juego de lenguaje wittgensteniano, donde si dice "si se comete A, se aplica la regla B", conociendo los conceptos de regla, de regla A y de regla $\mathrm{B}$, el accionar del sistema es mecánico. Es posible medir al fenómeno jurídico en esta descripción. Cuando se piensa en (2), la red de relaciones holísticas permite la explicación de un concepto, pero a la vez, se depende de otro, ello permite una cadena de comprobación que pasa por una justificación teórica, lo que luego da el pase a una explicación teórica que al mismo tiempo, se trasunta en la práctica. Por ejemplo, explicar el contenido de la imputación de responsabilidad puede hacerse a través de un cuerpo normativo, luego a la explicación de por qué se aplica una regla al caso en cuestión, lo que holísticamente deriva en que se imputó responsabilidad. (3) está de la mano con (2), aunque ello es para armar

33. ZIPURSKY, Benjamin: "Pragmatic Conceptualism”. En: Legal Theory, 6(4), 2000. Págs. 457-78.

34. ROSS, Alf: Sobre el derecho y la justicia. Eudeba, Buenos Aires, 1994 (trad. Genaro Carrió). Págs. 46-52.

Facultad de Derecho y Ciencias Sociales - Universidad de Valparaíso - Chile 
el argumento completo, al estilo del "barco de Neurath". (4) es muy práctico, y puede ser determinante a la hora de construir una teoría de la adjudicación. (5) parece ser la norma/argumento de clausura que esbozaba al inicio de la explicación de la tesis de Coleman sobre un pragmatismo jurídico, sobre la aceptabilidad de las creencias, hay algunas que no se examinan y otras que sí. Finalmente, se reduce a las “mejores explicaciones posibles" y por tanto, las más útiles.

El pragmatismo de Coleman, luego, si bien está de acuerdo en que el derecho es una práctica normativa (law as facts) es preciso distinguir que, cuando refiere a una práctica, apunta al componente fáctico de lo que ocurre en la realidad y que es preciso explicar de la mejor y más satisfactoria manera posible.

\section{PERSPECTIVA DE UN ENFOQUE CIENTÍFICO- PRAGMÁTICO EN LA TEORÍA JURÍDICA DEL SIGLO XXI}

"El punto esencial es el caracterizar un episodio o estado como uno de conocimiento... pues estamos ubicados en esta fase, en la lógica espacial de las razones, de las justificaciones, siendo totalmente capaces para justificar lo que uno dice" 35 . Según Bernstein, esta es justamente la verdad de los hechos que decimos saber, luego se requiere el indispensable compromiso de los valores y las normas, pues sin ellos no hay mundo y por tanto, no hay hechos ${ }^{36}$.

El pragmatismo jurídico de Coleman se inscribe como un conjunto de indicadores metódicos que deben otorgar una certeza y no dejar cabos sueltos. En el caso de la predictibilidad que la conjunción de las ciencias sociales con las ciencias naturales y en el caso de la revisión de las creencias, la consiliencia y un análisis funcional, no puede haber respuestas equivocadas ni ambiguas ni vagas ${ }^{37}$.

35. SELLARS, Wilfrid: Science, Perception and Reality. Harvard University Press, Cambridge (MA), 1997. Pág. 169.

36. BERNSTEIN, R. J. op. cit. Pág. 158.

37. Estas tesis no pueden explicar la totalidad de fenómenos que acometen al derecho, pueden ser efectivas para hacerse cargo de algunas áreas de lo jurídico,

Revista de Ciencias Sociales - Número 65 (2014) - Universidad de Valparaíso - ISSN 0716-7725-Valparaíso, Chile 
Este enfoque científico del derecho puede hacer ingreso a la disputa sobre los métodos y propósitos de la filosofía del derecho a partir del vacío que The Concept of Law dejó. El texto de Hart soslaya que los resultados de la reflexión filosófica sobre, el derecho pueden clasificarse sin distorsión como enunciados analíticos o sintéticos. Este autor adoptaría un postulado más pragmático, pero que a la hora de adaptarlo a su modelo de las reglas, fracasa. Pues la misión del proyecto hartiano, en última instancia, es elucidar las relaciones entre nuestros conceptos ${ }^{38}$ y nuestras distintas formas de hablar (normar), por lo mismo nuestro concepto de derecho está relacionado con nuestros conceptos de regla y de obligación ${ }^{39}$.

$\mathrm{Al}$ respecto, Ian Farrell agrega lo siguiente:

“(...) el análisis conceptual se limita a sacar conclusiones acerca de lo que es el concepto, esclareciendo la estructura subyacente del concepto y estableciendo si determinadas situaciones particulares son cubiertas por él (...) Esto implica típicamente una exposición de la concepción folk del concepto ${ }^{40}$. El análisis conceptual (...) no va más allá de esto. En particular, no formula

pero la insuficiencia cientificista se deja notar. En el caso de ambas teorías que pueden operar muy bien como teorías de la adjudicación; la pragmatista, así también en el caso de la justificación teórica de un enfoque que involucra al derecho, como el análisis económico del derecho. A favor de esto, consúltese la introducción en RATTI, Giovanni Battista: El Gobierno de las normas. Marcial Pons, Madrid, 2013.

38. Por otro lado, en un enfoque pragmatista como el de Coleman, se busca crear las relaciones entre conceptos, a partir de una red de relaciones holísticas.

39. RIFFO ELGUETA, Ernesto: Explicando el derecho. Pluralismo, prácticas e instituciones jurídicas. Tesis de pregrado. Facultad de Derecho, Universidad de Chile, 2012. Pag. 133.

40. Con esta expresión hace referencia a "lo popular". El concepto folk del derecho sería entonces una cuestión de vocabulario común y ordinario que está en boca de todos, prácticamente. Sobre él también, Shapiro respecto de los truisms about law (SHAPIRO, Scott J.: Legality. Harvard University Press, Cambridge (MA), 2011).

Facultad de Derecho y Ciencias Sociales - Universidad de Valparaíso - Chile 
ninguna tesis acerca de la naturaleza del universo. Es meramente descriptiva: y describe el concepto, no el mundo"41-42.

The Concept of Law en su propósito más general, dice su autor, es no dar un significado de la palabra derecho, sino que esclarecer sus probables usos. Entonces queda cooptado en su finalidad de ser de "sociología" descriptiva, pues no da cuenta de la sociedad en sí. Tiene una arista menos y es ahí donde teorías epistémicamente superiores como el pragmatismo jurídico de Coleman resulta mejor para analizar al fenómeno jurídico. En ese sentido, la misma falibilidad del ser humano resulta otro argumento, en tanto si dice Hart que son las prácticas humanas las que crean el derecho, ¿̇cómo él puede dar cuenta de esto?

Sobre ese punto, la idea de $e l$ concepto de derecho impone una generalidad y que el giro hermenéutico podría aseverar que este autor difumina el concepto folk y entonces, asume que todos los individuos de una colectividad manejan el lenguaje que el presupone. Los conceptos concomitantes a la noción de derecho o como yo puedo hablar del derecho se entienden asumidos. Aquí las cuestiones empíricas atacan, pues ¿̇ómo es efectivamente usado el concepto de derecho? ¿Representa ese concepto la estructura coherente supuestamente atisbada por Hart (y luego Raz)? Después del empirismo, y cuando llegan las certezas se está en una situación epistémica ideal ${ }^{43}$.

La posición de Coleman se ubica como una "teoría práctica del derecho" y vislumbra a su correlato en el aparataje decisionista de la judicatura estadounidense, una cuestión que se inició bajo la descripción que Oliver Wendell Holmes hizo en The Path of the Law (1897). Claro que sin un claro bagaje filosófico que pudiera sustentar la tesis más allá

41. Que el concepto obviamente pretende describir o al que, por lo menos, pretende hacer referencia.

42. FARRELL, Ian: "H.L.A. Hart and the Methodology of Jurisprudence". En: Texas Law Review, 84, 2006. Pág. 999.

43. PUTNAM, Hilary: Realism with a Human Face. Harvard University Press, Cambridge (MA), 1980. Pág. viii.

Revista de Ciencias Sociales - Número 65 (2014) - Universidad de Valparáíso - ISSN 0716-7725-Valparaíso, Chile 
de una explicación de la puesta en marcha del derecho. Este antecedente muestra que el pragmatismo tiene otra ramificación, no sólo circunscrita a la filosofía estadounidense de mano de los primeros pragmatistas, sino también, el realismo jurídico norteamericano, que también tiene sus versiones en los trabajos de entre otros, Richard Posner, Jerome Frank, Karl Llewellyn y Roscoe Pound ${ }^{44}$.

El análisis de los conceptos para entender al derecho no parece fructífero frente a estas posturas; la cita de Farrell parece lapidaria al respecto. No obstante, a 20 años del Postscript donde Hart responde a sus críticos antes de fallecer y a 53 de The Concept of Law, la reconstrucción de las discusiones a las que ambos textos dieron lugar, puede evidenciar la influencia de Hart que sin duda que sigue proliferando en la discusión erudita. Por lo mismo, interrogarse sobre el concepto de derecho será una cuestión que tendrá lugar en la literatura por un buen tiempo. A ello, las diferentes empresas por forjar una teoría del derecho y que se proclaman tales, tendrán que debatir contra las posturas de Hart. Sin embargo, el pragmatismo de Coleman se enfoca más como una teoría práctica del derecho, no interesada directamente en dar cuenta de "qué es el derecho", como concepto propiamente tal. Solo describe parte del mismo, en el caso de una teoría (descriptiva) de la adjudicación.

El pragmatismo jurídico se inserta en la discusión contemporánea como una postura perteneciente al análisis cientificista del derecho, pues se valen de medios rigurosos y de las ciencias naturales para reflexionar sobre lo jurídico. Ahora bien, ambas posturas no nacen directamente al alero de The Concept of Law, ello es interesante, pues como se dijo, la ramificación de esta vertiente reflexiva del derecho se remite al texto del juez Holmes. La raigambre realista se orienta hacia la creación de una teoría del derecho norteamericano. La separación con el iusrealismo escandinavo debe ser notoria, pues los problemas que acometen a la teoría norteamericana son radicalmente distintos ${ }^{45}$. Los iusrealistas

44. CARBONELL, Flavia: La idea de corrección en el derecho. Tesis doctoral. Departamento de Derecho Internacional, Eclesiástico y Filosofía del Derecho, Universidad Carlos III de Madrid, 2013. Pág. 400.

45. Ibíd. Págs. 402-10.

Facultad de Derecho y Ciencias Sociales - Universidad de Valparaíso - Chile 
norteamericanos inclusive, tuvieron una separación entre formalistas y escépticos, a lo cual, Hart respondióo ${ }^{46}$. Según Leiter, "para los iusrealistas, los casos son predecibles, en la medida que lo son, no porque las razones jurídicas están determinadas, sino porque una investigación sociocientífica puede identificar aquellos factores no jurídicos que realmente determinan el curso de la decisión" ${ }^{47}$. Al mismo tiempo, el otorgar una base más elaborada y que pueda ser conducente a cero errores, es la tarea de Leiter. Sobre ello, alega Moore lo siguiente: "según la reconstrucción de Leiter, los realistas jurídicos tenían sólidas bases naturalistas y pragmatistas para convertir la teoría jurídica en una ciencia social acerca del comportamiento judicial" ${ }^{\prime 8}$. Aunque como no eran doctos en filosofía ni tampoco tenían comprensión de las discusiones, como por ejemplo, el debate "Quine-Putnam”, les era difícil. Greenberg, agrega que el refuerzo entregado por Leiter a los realistas, a partir de la propuesta de naturalización de la filosofía del derecho, no es más que la evidencia de que el proyecto por explicar el derecho por parte de los iusrealistas, fue un fracaso ${ }^{49}$. Para Leiter, la "cientifización" del enfoque iusrealista no es más que el tratamiento de la cuestión del derecho como una cuestión empírica a ser estudiada científicamente ${ }^{50}$.

La teoría del derecho norteamericana, soslayó el proyecto normativista de Kelsen, es más, como el régimen jurídico propio es distinto del explicado por el austriaco, no se tuvo a esa postura como antecedente filosófico ${ }^{51}$. Tampoco se tiene en cuenta el trabajo de Ross

46. HART, H. L. A.: "American Jurisprudence through English Eyes: The Nightmare and The Noble Dream”. En: Georgia Law Review, 11, 1977. Págs. 969-89.

47. LEITER, B. Naturalismo y teoría ... ob. cit. Pág. 139.

48. MOORE, Michael: Educating Oneself in Public: Critical Essays in Jurisprudence. Oxford University Press, Oxford, 2000. Pág. 33.

49. GREENBERG, Mike: "Naturalism in Epistemology and the Philosophy of Law, Parts I and II”. En: Law and Philosophy, 30, 2011. Págs. 419-76.

50. LEITER, B. Naturalismo y teoría ... ob. cit. Pág. 168.

51. TAMANAHA, Brian: "Understanding Legal Realism”. En: Texas Law Review, 87(4), 2008. Págs. 731-86.

Revista de Ciencias Sociales - Número 65 (2014) - Universidad de Valparáíso - ISSN 0716-7725-Valparaíso, Chile 
y los demás iusrealistas escandinavos que se posicionan en otra vereda, ¿qué decir de los iusrealistas genoveses ${ }^{52}$, una corriente que se ha asomado fuertemente en los últimos años como (otra) "teoría analítica del derecho"? 53

\section{ALGUNAS CONCLUSIONES}

$\mathrm{Ni}$ en el iusrealismo norteamericano ni en el pragmatismo jurídico de Coleman existe una pretensión notoria de construir una teoría del derecho tan exhaustiva (y general) como la que crearon Kelsen o Hart, sin embargo, como tesis que apoyan el análisis cientificista del derecho quedan de manifiesto ${ }^{54}$. El pragmatismo jurídico de Coleman se entiende, en definitiva, como una explicación del derecho en base a las distintas áreas que regula en mira a que el derecho es una práctica social, útil para los propósitos humanos. El derecho es funcional a la vida humana y como tal, debe tener justificaciones teóricas y explicaciones teóricas.

El proyecto de naturalización de la filosofía de Quine y los trabajos de Putnam y Rorty permitieron a la concepción cientificista subsistir más allá de la gloria que el positivismo lógico había alcanzado en la primera mitad del siglo XX. La recepción de la tesis de Coleman para aplicarlas en los conocimientos iusfilosóficos en que fueron formados, fue fundamental para encontrar dos cosas:

52. LEITER, Brian: "Legal Realisms, Old and New". En: Valparaiso University Law Review, 48, 2013, en prensa; FERRER, Jordi y BATTISTA, Giovanni (eds.): El Realismo Jurídico Genovés. Marcial Pons, Madrid, 2011.

53. COMANDUCCI, Paolo: Hacia una teoría analítica del derecho. Ensayos escogidos. Centro de Estudios Políticos y Constitucionales, Madrid, 2010; ESCUDERO, Rafael: "Estudio preliminar". En: Comanducci, Paolo: Hacia una teoría analítica del derecho. Ensayos escogidos. Centro de Estudios Políticos y Constitucionales, Madrid, 2010. Pág. xv-li.

54. Leiter explicaba que las tesis del análisis conceptual son siempre vulnerables a los requerimientos de la construcción teórica a posteriori. Es extraño que los filósofos sigan tranquilamente utilizando el análisis conceptual, considerando la serie desastrosamente mala de seudoverdades que se han alcanzado con sus métodos LEITER, B. Naturalismo y teoría... ob. cit. Pág. 188.

Facultad de Derecho y Ciencias Sociales - Universidad de Valparaíso - Chile 
En primer lugar, La evolución de la teoría jurídica norteamericana basada en la predicción de la acción tribunalicia, el trabajo de la judicatura y el funcionamiento de las reglas, llamada también iusrealismo norteamericano, hacia una versión justificada y explicada teórica y filosóficamente con los antecedentes de un conjunto de trabajos que se desarrollaron en Estados Unidos a lo largo del siglo $\mathrm{XX}$ y que para completarla, con el fin de realmente otorgar el conocimiento de la realidad en que el derecho estaba inmerso, se sometió a la metodología que mezclaba las ciencias sociales con las ciencias naturales.

En segundo, la continuidad de los movimientos teórico-jurídicos que se proyectó en Estados Unidos durante el siglo XX. La mayoría de los expertos en la materia o bien, quienes teorizaban al derecho en términos de posibilitar su explicación, no eran doctos en filosofía, probablemente habían leído a C. S. Pierce, por entonces, uno de los referentes en el pragmatismo, etiqueta que tanto se aplicaba a los referentes del realismo jurídico norteamericano. Es más, vemos que Haack se pregunta si The Path of the Law entrega herramientas pragmatistas para el análisis del derecho (2012). El proyecto de Coleman, con el afán de hacer valer realmente la etiqueta de pragmatista a la reflexión del derecho, intentó justificar y explicar la institución del derecho de daños (tort law). The Practice of Principle, ergo, se transformó en la última década en un texto de consulta en el ámbito del Law and Economics, pues el bagaje que incluye los criterios pragmáticos (coherencia, simplicidad y consiliencia) es susceptible de ser utilizada en las otras áreas o ramas del derecho. Asimismo, la extensión de un método para concebir una teoría práctica del derecho funciona para los demás movimientos que han nacido en Estados Unidos en las distintas facultades de derecho y que han sido, sindicados como intentos por teorizar a lo jurídico, de ellos, cabe mencionar al grupo de los Critical Legal Studies (CLS). La sencillez y a la vez, rigurosidad (revisionista, inclusive) en la confirmación de las creencias, enunciados y estructura del derecho, y del intento por teorizar al fenómeno jurídico, junto con la importancia de crear soluciones prácticas y útiles a las cuestiones que puede suscitar la aplicación de reglas, es lo principal que destaco del enfoque pragmatista de Coleman. Ello, en definitiva, la sitúa como un enfoque científico en la teoría del derecho de este siglo.

Revista de Ciencias Sociales - Número 65 (2014) - Universidad de Valparáíso - ISSN 0716-7725-Valparaíso, Chile 


\section{REFERENCIAS}

BACON, Michael: Pragmatism. Polity Press, Oxford, 2012.

BERNSTEIN, Richard J.: The Pragmatic Turn. Cambridge University Press, Cambridge, 2010.

CARBONELL, Flavia: La idea de corrección en el derecho. Tesis doctoral. Departamento de Derecho Internacional, Eclesiástico y Filosofía

del Derecho, Universidad Carlos III de Madrid, 2013.

COLEMAN, Jules: "Los fundamentos del bienestar". En: Derecho y Humanidades, 10, 2004. Págs. 17-46.

COLEMAN, Jules: The Practice of Principle. In Defence of a Pragmatist Approach to Legal Theory. Oxford University Press, Oxford, 2001.

COLEMAN, Jules: Risks and Wrongs. Cambridge University Press, Cambridge, 1992.

COLEMAN, Jules: "Negative and Positive Positivism”. En: Cohen, M. (ed.): Ronald Dworkin and Contemporary Jurisprudence.

Duckworth, Londres, 1983. Págs. 28-48.

COMANDUCCI, Paolo: Hacia una teoría analítica del derecho. Ensayos escogidos. Centro de Estudios Políticos y Constitucionales, Madrid, 2010.

DWORKIN, Ronald: Taking Rights Seriously. Harvard University Press, Cambridge (MA), 1977.

ESCUDERO, Rafael: "Estudio preliminar". En: Comanducci, Paolo: Hacia una teoría analítica del derecho. Ensayos escogidos. Centro de Estudios Políticos y Constitucionales, Madrid, 2010. Págs. xv-li.

FARRELL, Ian: "H.L.A. Hart and the Methodology of Jurisprudence". En: Texas Law Review, 84, 2006. Págs. 983-1011.

FERRER, Jordi y BATTISTA, Giovanni (eds.): El Realismo Jurídico Genovés. Marcial Pons, Madrid, 2011.

GREENBERG, Mike: "Naturalism in Epistemology and the Philosophy of Law, Parts I and II". En: Law and Philosophy, 30, 2011. Págs. 419-76.

HAACK, Susan: "On Legal Pragmatism: Where Does "The Path of the Law" Lead Us?". En: Pragmatism Today. The Journal of the

Central-European Pragmatism Forum, 3(1), 2012. Págs. 8-31.

HAACK, Susan: Pragmatism: Old and New. Prometheus Books, Nueva York, 2006.

Facultad de Derecho y Ciencias Sociales - Universidad de Valparaíso - Chile 
HAACK, Susan: "Defendiendo la ciencia, dentro de la razón". En: Contrastes, Revista Interdisciplinar de Filosofía, Suplemento 3, 1998. Págs. 37-56.

HAACK, Susan: "Pragmatism". En: Dancy, J. y Sosa, E. (eds.): $A$ Companion to Epistemology. Blackwell, Oxford, 1992.

Págs. 351-7.

HART, H. L. A.: "American Jurisprudence through English Eyes: The Nightmare and The Noble Dream". En: Georgia Law Review, 11, 1977. Págs. 969-89.

HART, H. L. A.: The Concept of Law. Clarendon Press, Oxford, 1961.

HOLMES, O. W.: "The Path of the Law". En: Harvard Law Review, 10, 1897. Págs. 457-78.

HOOKWAY, Christopher: "Pragmatism", The Stanford Encyclopedia of Philosophy, 2008. Disponible en: http://plato.stanford.edu/entries/ pragmatism/ Fecha última consulta: 18 de marzo de 2014.

JAMES, William: Pragmatism. Hackett Publishing, Indianapolis (IN), 1977.

LEITER, Brian: "Legal Realisms, Old and New”. En: Valparaiso University Law Review, 48, 2013, en prensa.

LEITER, Brian: Naturalismo y teoría del derecho. Marcial Pons, Madrid, 2012 (trad. Giovanni Battista Ratti).

LÓPEZ PÉREZ, Nicolás: "El naturalismo jurídico como teoría del derecho: red filosófica y conceptual de un cientificismo revisitado”. En: Derecho y Humanidades, 22, 2013, en prensa.

MACKIE, J. L. "The Third Theory of Law". En: Cohen, M. (ed.): Ronald Dworkin and Contemporary Jurisprudence. Duckworth, Londres, 1983. Págs. 161-70.

MISAK, Cheryl (ed.): New Pragmatists. Oxford University Press, Oxford, 2009.

MOORE, Michael: Educating Oneself in Public: Critical Essays in Jurisprudence. Oxford University Press, Oxford, 2000.

ORELLANA BENADO, M. E.: "Tradiciones y concepciones en filosofía”. En: NUDLER, O. (ed.): Filosofía de la Filosofía.

Enciclopedia Iberoamericana de Filosofía, 31. Trotta, Madrid, 2010. Págs. 49-78.

PEIRCE, C. S.: The Essential Peirce (eds. Houser, N. y Kloerel, C.). Indiana University Press, Bloomington (IN), 1992. 
PUTNAM, Hilary: El desplome de la dicotomía hecho-valor y otros ensayos. Paidós, Barcelona, 2001 (trad. Francesc Forn i Argimon).

PUTNAM, Hilary: Realism with a Human Face. Harvard University Press, Cambridge (MA), 1980.

QUINE, W.V.: The Ways of Paradox and Other Essays. Harvard University Press, Cambridge (MA), 1976.

RATTI, Giovanni Battista: El Gobierno de las normas. Marcial Pons, Madrid, 2013.

RESCHER, Nicholas. Realistic Pragmatism: An introduction to pragmatic philosophy. State University of New York, Nueva York, 2000.

RIFFO ELGUETA, Ernesto: Explicando el derecho. Pluralismo, prácticas e instituciones jurídicas. Tesis de pregrado. Facultad de Derecho, Universidad de Chile, 2012.

ROSS, Alf: Sobre el derecho y la justicia. Eudeba, Buenos Aires, 1994 (trad. Genaro Carrió).

SELLARS, Wilfrid: Science, Perception and Reality. Harvard University Press, Cambridge (MA), 1997.

SHAPIRO, Scott J.: Legality. Harvard University Press, Cambridge (MA), 2011.

SINGER, Joseph: "Legal Realism Now”. En: California Law Review, 76, 1988. Págs. 475-95.

TAMANAHA, Brian: “Understanding Legal Realism”. En: Texas Law Review, 87(4), 2008. Págs. 731-86.

THAGARD, P.: "The Best Explanation: Criteria for Theory Choice". En: Journal of Philosophy, 75(2), 1978. Págs. 76-92.

WARNER, R.: "Why Pragmatism? The Puzzling Place of Pragmatism in Critical Theory". En: University of Illinois Law Review, 1993.

Págs. 535-63.

ZIPURSKY, Benjamin: "Pragmatism, Positivism, and the Conventionalistic Fallacy”. En: Campbell, J. K., O’Rourke, M.

y Shier, D. (eds.): Law and Social Justice. The MIT Press, Cambridge (MA), 2005.

ZIPURSKY, Benjamin: "Pragmatic Conceptualism”. En: Legal Theory, 6(4), 2000. Págs. 457-78.

Facultad de Derecho y Ciencias Sociales - Universidad de Valparaíso - Chile 